\title{
ISOLATION OF A Y CHROMOSOMAL DNA SEQUENCE AND ITS CLINICAL APPLICATION
}

\author{
Masato Tsukahara, ${ }^{1}$ Shinya Matsuura, ${ }^{1}$ Fumio Kishi, ${ }^{1}$ \\ Akira Yoshida, ${ }^{2}$ and Tadashi KaJII ${ }^{1}$ \\ ${ }^{1}$ Department of Pediatrics, Yamaguchi University School of Medicine, \\ Ube 755, Japan \\ 2Department of Biochemical Genetics, Beckman Research Institute of \\ the City of Hope, Duarte, California 91010
}

\begin{abstract}
Summary A $4.6 \mathrm{~kb}$ long, Y-specific DNA fragment was isolated from a flow-sorted human $Y$ chromosomal library, and its male specificity was confirmed by Southern blot analysis. The fragment, designated as pY-80, was proven with an in situ hybridization experiment to have originated from the Yp11.2-Ypter region. Its 2,808 bp section was sequenced. The polymerase chain reaction proceeded with oligonucleotides flanking a 666 bp Pst I-EcoRI fragment of the sequence as primers and a male genomic DNA as a template, but not with a female genomic DNA. Preliminary tests of samples of various sources successfully detected the Y-specific fragment in male-derived samples, including mouth wash, single hair roots, urinary epithelial cells, dried blood spots and amniotic fluid cells.
\end{abstract}

Key Words human $\mathrm{Y}$ specific DNA sequences, polymerase chain reaction, clinical application

\section{INTRODUCTION}

The human $\mathrm{Y}$ chromosome consists mainly of the following three regions: 1) the "pseudoautosomal," $X$ and Y-pairing region at $Y p 11.3$; 2) the pericentromeric, $\mathrm{X}$ and $\mathrm{Y}$-non-paring region; and 3 ) the heterochromatic, non-pairing region at $\mathrm{Yq12}$. Y specific DNA probes from these regions are useful for various purposes including 1) prenatal diagnosis of sex-linked disorders, 2) following up the hostversus-graft cells in patients with bone marrow transplants from the opposite sex, and 3) analyses of sex chromosomal disorders such as a Y/autosome translocation, non-fluorescent $\mathrm{Y}$ chromosome, $\mathrm{Yq}$ isochromosome, and $\mathrm{XX}$ males. While a number of Y-specific DNA fragments have been cloned, few of them have been

Received September 28, 1990; revised version received October 29, 1990; Accepted November 20, 1990. 
sequenced (Cooke et al., 1984; Nakahori et al., 1986; Arnemann et al., 1987; Ellis et al., 1989; Sinclair et al., 1990).

We describe here a $Y$-specific clone, located on the short arm of the $Y$ chromosome, and its DNA sequence. Its clinical application is also discussed.

\section{MATERIALS AND METHODS}

$Y$ chromosome-specific library. A human Y chromosome-specific library was provided by American Type Culture Collection (ATCC No. 57715).

Screening of the human $Y$ chromosome-specific library. The library was screened by the plaque hybridization method with a HindIII-digested female genomic DNA as a probe. Phage DNAs from the plaques that did not hybridize to the human female genomic DNA were prepared by the plate lysis method.

Isolation of Y-specific DNA. The isolated phage DNAs were digested with HindIII, electrophoresed on $0.6 \%$ agarose gel, and Southern blotting was carried out using HindIII-cleaved, ${ }^{32} \mathrm{P}-\mathrm{labeled}$ human female and male genomic DNAs as a probe. The DNA clones hybridized to the male DNA probe but not to the female probe were further selected, and inserts were separated on $0.8 \%$ low melting agarose gel. The selected inserts were subcloned into pUC118.

$D N A$ sequence analysis. Suitable restriction fragments were subcloned into M13mp18 and mp19 vectors and sequenced by the dideoxy method, using an M13 sequencing primer.

Chromosomal assignment. Chromosomal localization of the cloned Y-specific DNA was determined by in situ hybridization (Zabel et al., 1983). The DNA probe was labeled with $\left[{ }^{3} \mathrm{H}\right] \mathrm{dCTP},\left[{ }^{3} \mathrm{H}\right] \mathrm{dATP}$, and $\left[{ }^{3} \mathrm{H}\right] \mathrm{dTTP}$ to a specinc activity of $1.1 \times 10^{7} \mathrm{cpm} / \mu \mathrm{g}$ by random primer labeling. Hybridized slides were dipped into Kodak nuclear track emulsion (NTB II), and exposed at $4^{\circ} \mathrm{C}$ for 10 days. After the autoradiography, the slides were stained using the Hoechst-Giemsa method as described previously (Tsukahara and Kajii, 1985).

DNA extraction from various samples. Peripheral blood DNA was extracted from 6 normal subjects ( 3 males and 3 females) by the method of Wyman and White (1980). Buccal epithelial cells ( 3 males and 4 females) were obtained by centrifugation from mouth wash with $15 \mathrm{ml}$ of saline and urinary epithelial cells (4 males and 4 females) from $15 \mathrm{ml}$ of urine each, and washed with $1 \mathrm{ml}$ of Tris-buffered saline. A single hair was plucked each from 6 individuals ( 3 males and 3 females) and cut with scissors to obtain the hair root. Amniotic fluid cells from 3 pregnant women were collected by centrifugation of amniotic fluid $(1.5 \mathrm{ml})$. The cells thus collected were suspended in $400 \mu \mathrm{l}$ of lysis buffer $(0.32 \mathrm{M}$ sucrose, $10 \mathrm{mM}$ Tris- $\mathrm{HCl}$ $\mathrm{pH} 7.5,5 \mathrm{mM} \mathrm{MgCl}_{2}, 1 \%$ Triton X-100) containing $200 \mu \mathrm{g}$ of proteinase $\mathrm{K}$ and $0.5 \%$ sodium dodecyl sulfate. The cell suspension was incubated for $2 \mathrm{hr}$ at $65^{\circ} \mathrm{C}$, followed by phenol/chloroform and chloroform extractions. DNA was then precipitated with ethanol and redissolved in 25,4 of sterile water and its $8 \mu$ portion 
was used for the polymerase chain reaction (PCR).

Dried blood spots on a filter paper from 5 newborn babies ( 3 males and 2 females) were obtained. A $1 \mathrm{~cm}$ dried blood spot was cut out from each sample and rehydrated by continuously shaking in $2 \mathrm{ml}$ of Tris-buffered saline containing 200 $\mu \mathrm{g}$ of proteinase $\mathrm{K}$ for $4 \mathrm{hr}$. Cells were centrifuged and resuspended in $50 \mu \mathrm{l}$ of sterile water and boiled for $10 \mathrm{~min}$. Cellular debris were then pelleted by centrifugation for $5 \mathrm{~min}$ and the supernatant was used directly for PCR amplification.

Polymerase chain reaction. The oligonucleotides were synthesized on an Applied Biosystem 381A DNA synthesizer and purified by polyacrylamide gel electrophoresis. The reaction mixture consisted of $50 \mathrm{mM} \mathrm{KCl}, 10 \mathrm{mM}$ Tris- $\mathrm{HCl}$, $\mathrm{pH} 8.3,1.5 \mathrm{mM} \mathrm{MgCl}, 0.01 \%$ gelatin, 25 pmol of each primer, $0.2 \mathrm{~mm}$ of each of the four deoxynucleotide triphosphates (dATP, dCTP, dTTP, and dGTP), 2.5 units of Taq DNA polymerase and the sample, for a total volume of $25 \mu \mathrm{l}$. The amplification reaction was performed in a programmed temperature control system (PC-600, Astec Co, Fukuoka). The samples were subjected to 30 cycles of denaturation at $94^{\circ} \mathrm{C}$ for $1.5 \mathrm{~min}$, annealing at $50^{\circ} \mathrm{C}$ for $2 \mathrm{~min}$ and extension at $70^{\circ} \mathrm{C}$ for $3 \mathrm{~min}$. The amplified product was analyzed on $1.5 \%$ agarose gel electrophoresis and transferred onto a nylon membrane.

\section{RESULTS}

\section{Screening of $Y$ chromosome library and isolation of $Y$-specific DNA fragments}

Of the $3 \times 10^{6}$ recombinant phage particles, 186 plaques that did not hybridize to the human female genomic DNA were obtained. Of the 186 plaques, 9 clones hybridized to male genomic DNA but not to female gemonic DNA (Table 1). Southern blot hybridization of these 9 clones with total female and male DNAs gave a male-specific banding pattern under both low and high stringent washing conditions. Clone pY-80 hybridized with a predominant fragment of $4.6 \mathrm{~kb}$, and in addition with two bands of weaker intensity, 3 and $2 \mathrm{~kb}$, respectively (Fig. 1,

Table 1. Y-specific DNA clones.

\begin{tabular}{lccc}
\hline Clone & $\begin{array}{c}\text { Clone } \\
\text { size }(\mathrm{kb})\end{array}$ & $\begin{array}{c}\text { Y-specific HindIII } \\
\text { fragments }(\mathrm{kb})\end{array}$ & $\begin{array}{c}\text { X and/or autosome } \\
\text { sequences }\end{array}$ \\
\hline pY-80 & 4.6 & $4.6 ; 3.0 ; 2.0$ & - \\
pY-25 & 4.8 & $7.2 ; 4.6$ & - \\
pY-48 & 5.0 & $5.4 ; 5.0$ & + \\
pY-50 & 4.4 & 12.0 & + \\
pY-102 & 5.2 & $5.6 ; 5.1$ & + \\
pY-111 & 5.2 & 4.6 & + \\
pY-146 & 4.2 & $7.0 ; 3.6$ & + \\
pY-148 & 5.0 & 6.6 & + \\
pY-153 & 3.6 & 3.1 & + \\
\hline
\end{tabular}

2. $\mathrm{X}$ and/or autosome sequences are shown as several bands or smear patterns common to female and male DNA.

Vol. 35, No. 4, 1990 
a and b). Clone pY-25 hybridized with two Y-specific fragments of 7.2 and 4.6 $\mathrm{kb}$. The other seven clones (pY-48, 50, 102, 111, 146, 148, and 153) each hybridized with $\mathrm{Y}$-specific fragments of various sizes, and in addition, with $\mathrm{X}$ and/or autosome sequences as shown as band or smear patterns common to both female and male genomic DNAs (Fig. 1c, Table 1). Of these 9 clones, pY-80 was further characterized.

Restriction map and nucleotide sequence of $p Y-80$

Figure 2 shows the restriction map of $\mathrm{pY}-80$ and its nucleotide sequencing

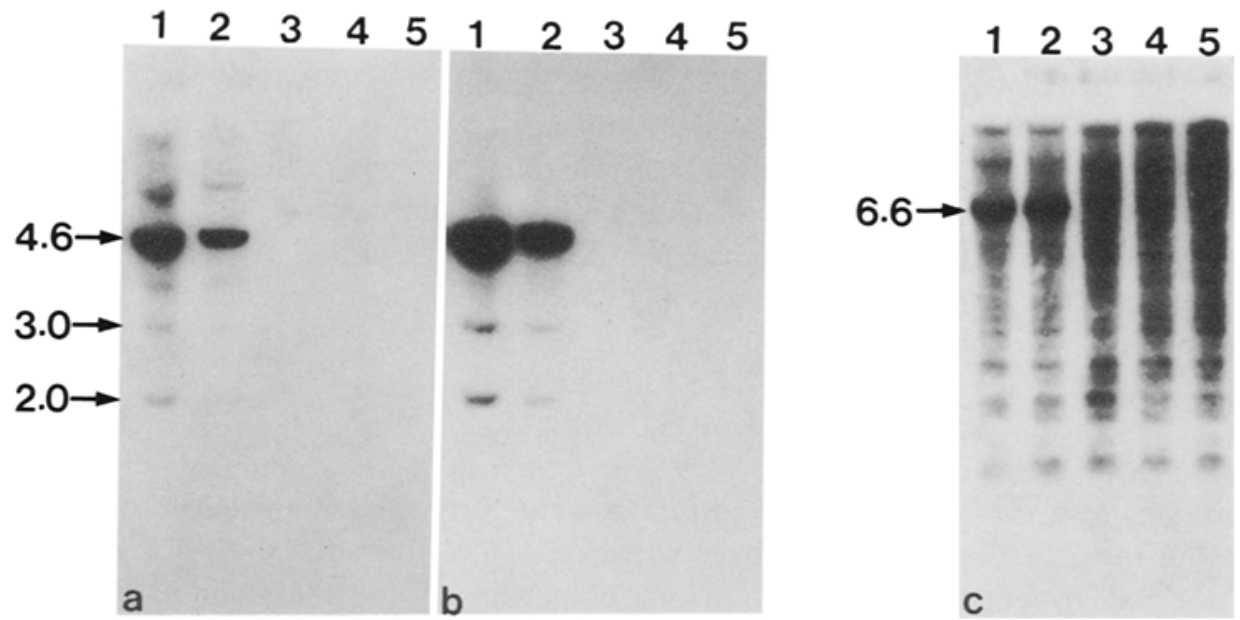

Fig. 1. Hybridization of clones pY-80 and pY-148 to HindIII-digested DNAs from males (lanes 1,2) and females (lanes 3-5). Hybridization of clone pY-80 under a) a low-stringent $\left(65^{\circ} \mathrm{C}, 2 \times \mathrm{SSPE}, 0.1 \% \mathrm{SDS}\right)$, and b) a high stringent $\left(65^{\circ} \mathrm{C}, 0.1 \times\right.$ SSPE, $0.1 \%$ SDS) washing conditions. c) Hybridization of clone pY-148. $Y$-specific bands are marked with arrows.

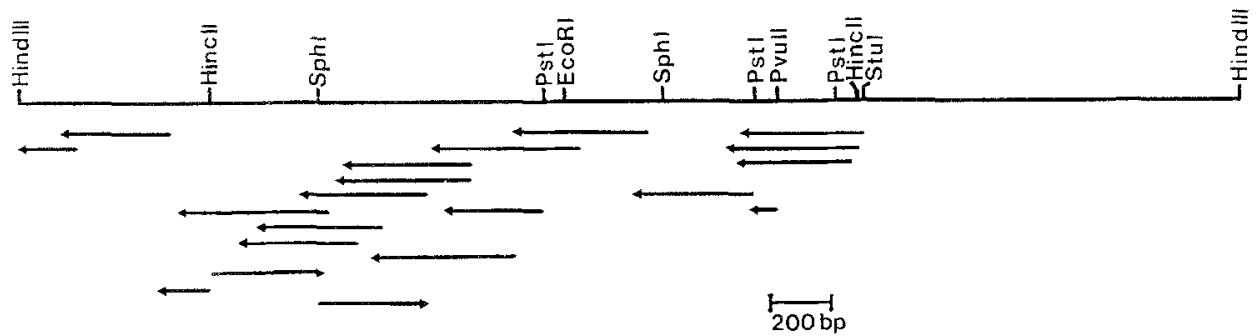

Fig. 2. Restriction map of pY-80 and sequence determination strategy. Horizontal arrows indicate the direction and extent of the sequencing.

Fig. 3. Nucleotide sequence of pY-80. Oligonucleotides used in the polymerase chain reaction are shown by arrows underlying the sequence. The nucleotide sequence has been submitted to the EMBL Data Library with accession number $\times 51582$. 
5. SLGLCTGTCAACCCCCAGCCCrGGGCTGCTTCCCTGGCCTCTTCTCTGTTCCCTCTCTCAGCGCOTA 70 AOGCCTGT CAACCCCCAGCCCPGGGCTGCTTCCCTGGCCTCTTCTCTGTTCCCTCTCTGAGGGCCTAACT CCCTTGGGTAGTGCTGCAGAATATAGAGCCACAGGCCCTGGCTCATGATCTGGTGGACTGGGCAAATTOG TCGTGACAGGTCAGGTTCTGGTTCAAAGCCAATTCCTCCGATGCCAAGGAATGTCGAAGAAGGTCCTTTG CCATGATGCCCCATAGCTGCCCCACCTCAGCAATCGTGCCGTAACCTGGGCCCTCACAGTCAGACANCCA GCTGAAGAAGCTCAGGCAGTGACCTGCGGGAAACTCGGGCTTTCACCTGCATGACCCTAGAACCACTGGA $P_{S t I}$
CTGCAGTGGAGCCAGTCGCCCTGTATCCTGGAGGGaGACGAGTCAGGAAGGGCACGCAGGCCCAGC'TCC CGAGGTACTACCCCCTCTACTCCTCAGgGaGGATGCCAACGCAATACTCCTTAGTCGTCACTTTGTT'TCC CGAGTACTACCCCCTCTACTCCICAGGGGGATGCCACCCAATACTCCTTAGTCGTCACTTTGT 560 CTGCCTGTCCAAGAAGGAGAAACAGGGCTGTGAAGGGGCAATTTCATCTAGGTGGGCTGAGGTGGCATTC TAGCCGGGGTGAAGCATGCGTTTCCCCTTCCCAGCTTTCCCGCTGAGACACACCTGAGCCCCAGAAGGAC CTCAACCTGACCAGGACCTTAGCACCCTCCCCAGACCCAGGCTTTCCATCCTGACCTGCAAATCCAACAT GCAGCT 'STGAAGGACTTTCTCATGGTTTCTGAGCTCCTTGCTCTCACCAGAAAGAATCAGAACTTTTAAA GTGTTCTTTATGCCAACTTAAATTTTCATTTTTACTACCTCATGTTTTGGATGAGGCATGTAT'TTTTAA A'TTTATTTTCACCTTATTGTACCTCTATGATAAACTGCTTGCTTACATTCATACCGTAATTATCTCTCAG

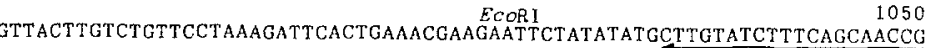
TATGTCAGATAGCACTGCACATTACTGCAGACATCGCATATACAGGTCCAAAGgTAGAGgaAgaAgaAGa AAGCAAGCGTTAAGCTCTATACATTCCTAAAAGCATATCAGAAACTCACAAATAACAGTGAAATCAAAGA

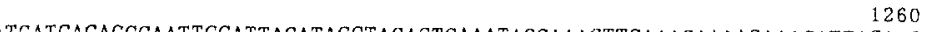
ATGATCACAGCCAATTCCATTACATACCTAGACTGAAATACGAANCTTCAAAGAAAAGAAACATTAGAAC TTIGGOT.TGTAAAAATTTCCTATAT 1330 TIGGGTTTGTAAAAATTTTCCTATATAGATAAAATTATTGGTAMCTGTGTCTCACTAGAAAACGTAAAC AAAAGTCCATGTTT TTCATATTTGTAAATATACATAGTTTTATTTCCATCAGTTATGACATGCAAGCAAG 1470

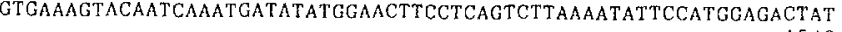
CAATTTTATGAAAACTATAAAGAATGCTTCATGAAACTACATTGTACAGTGCCATTTACTATTTTACTGA CATTT TAAATAATCAACAATTAAAGGGAATACATCAACATTATTTAATACCAATAACGSTATTTTTCTTG AGTAATCCTGTTGAAATTAAGGAT'TTAAATANAACATT AAAAACAAATTATATTGACTGATTTCAGCTT TGGATGAAATCATACTTGTGTATTTGTAGTAATGCGAAGCATAACTTTCTCCTCACAATTAATCTTTTAT

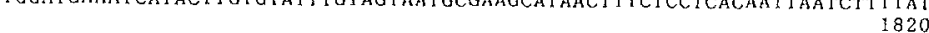
AACATCGGTGTTATAGTTTTCTCTGACACCAACATTGTGATATCGCACAGGTTTACTGCATGCATGCATT ACATGCCTCCAGAGAGTAGGCTTCAAATATATGGAAAAATTATATT TATGAAAAAATTCTAGGAAAGGGA ATGGTGAAATGGAAGAGAATTTCTCACTTGCTAACTGTTGGACATGGATTTGTATATATTTGGATATAG CACATACTGGCACACTGTGAGTTTGCCCATGTA'TATATACACTTATATGAGAAACCCATAATATATGGGT TGTGTAATCTTYTAATTAATCCATAATTGTATGTGTGTGAAATTAGATAAGCGGTTACCTTTTCTTTACT 2170
CAATTTGATGGAAAGCCAAAAAACTCTGTCCACCTTCATTTCAATTAATCCAATACTGTTAACTGCT AGCTTCATTCTCCTT GTTCTCTTACGGCAACCGGAAAGTTAATTCTCGCTCTAATTTGGCTTTCAAGGTO CGATCAACAAGAGTGTCACCTTGC'TGTGGATTGTGACCTCTGACTCCACCTCTGTCTTCCTTTTGCAGTO CTACCTTTGCATAGGTAACAAACTTTGTACATGGTTAAAAGGATAAAAGTTCAGTGAAATGTCAAGCCAT GCTGTGAAATGTTCCATAGT TTCTATATCTCTAATTGTCCTTTGATGTTATAGAGGCAAGAAAAATAATT CAATGTTTTTCTTAGTATCTAGTCCAATGCACTCTTTCTTCATAATACTGCAAACAAGGCACTGACATGG AAACGTGGCT'GGACGTCTCAAAATCTCTTCTCATTAATTACCATTATGTTAATCACTGTTGCCCACAACT 2660
GGAATTGGACTTTGAAATCCCCTGGTGGAAATTGCTATAATGGCTCAAACTACTGGAAAGACTATCTTTT TTTACCTCAMAATATCTGATGAGCATAGAOGT 2730 CCATCACTGCCTACTCAATAATAGGTATCCCAAACCTTTGAGCCAAACTGAGCTCAGGTGCTCCCACAAA Fig. 3. CCAACCTT 3 ,

Vol. 35, No. 4, 1990 
strategy. Figure 3 shows the nucleotide sequence of the 2,808 bp section of pY-80 spanning the StuI through HindIII sites. The GC content of the sequence was $40.2 \%$. The sequence revealed no homology with other Y-specific DNA sequences previously described (Cooke et al., 1984; Nakahori et al., 1986; Arnemann et al., 1987; Ellis et al., 1989; Sinclair et al., 1990).

\section{Chromosomal assignment of $p Y-80$}

From a chromosomally normal male, 21 metaphases with well-banded chromosomes were analyzed for in situ hybridization (Fig. 4). Overall, 60 grains were observed, of which $29(48 \%)$ were located on the $Y$ chromosome. Of the 29 grains, $25(86 \%)$ were localized at Yp11.2-Ypter.

PCR of $p Y-80$

A flanking site of PstI (nucleotide position, 351)-EcoRI (nucleotide position, 1017) fragment of pY- 80 was used for the PCR. The oligomers used were Y1; 5'-ATGACCCTAGAACCACTGGA-3' (upstream primer) and Y2; 5'-CGGTTGCTGAAAGATACAAG-3' (downstream primer). The oligomers span a 719 bp section of pY-80 (Fig. 3). Electrophoretic analysis of the PCR products revealed a $0.7 \mathrm{~kb}$ band with male specificity (Fig. 5a). Southern blot analysis of the gel was performed with a ${ }^{32} \mathrm{P}$-labeled Pst I-EcoRI fragment of $\mathrm{pY}-80$ to confirm the origin of the amplified product. The 719-bp amplified products were hybridized

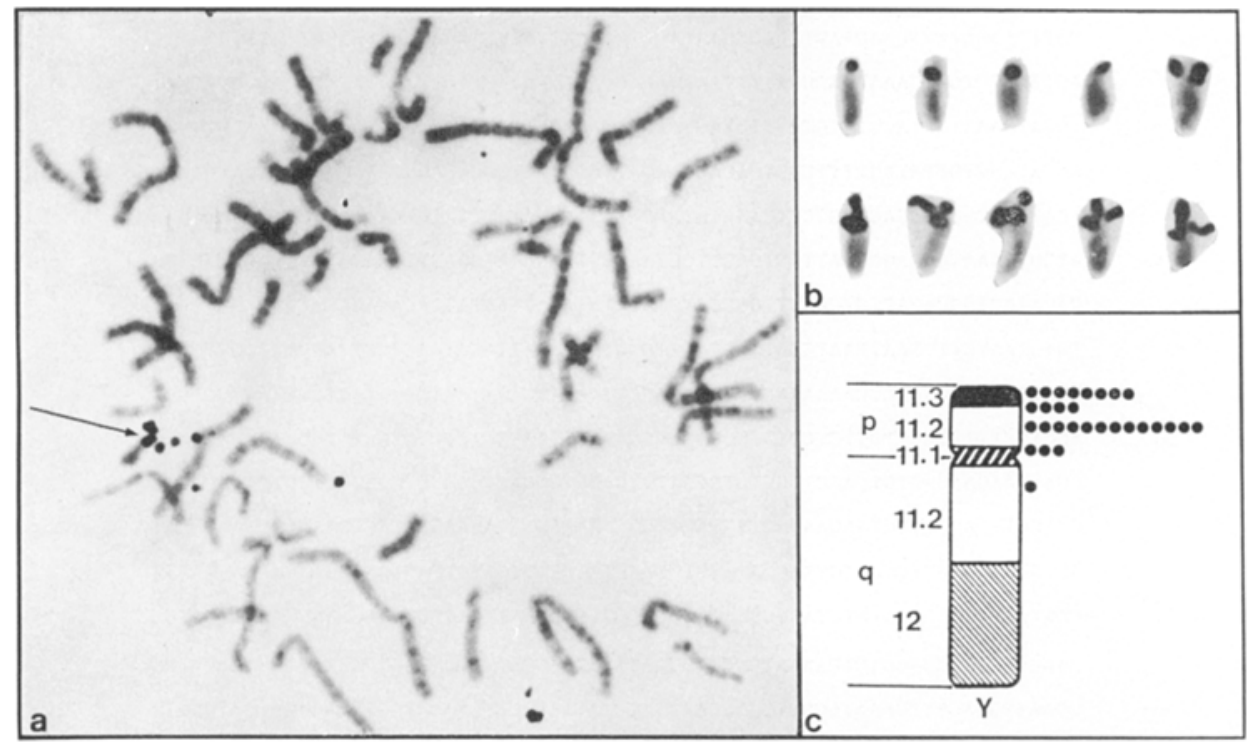

Fig. 4. a: Lebeling of the short arm of the $Y$ chromosome (arrow). b: Representative $Y$ chromosome with labeling. $c$ : Regional distribution of grains on the $Y$ chromosome. 


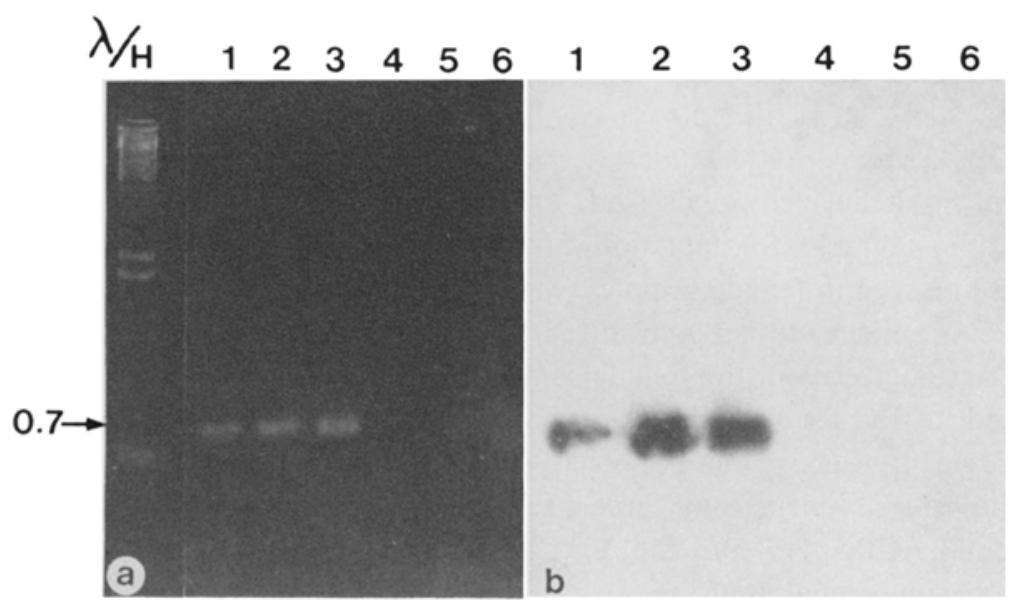

Fig. 5. a: Electrophoretic analysis of PCR products from males (lanes 1-3) and females (lanes 4-6). b: Southern blot analysis of the gel with a ${ }^{32}$ P-labeled Pst I-EcoRI fragment probe. The size of the PCR product is marked in $\mathrm{kb}$.

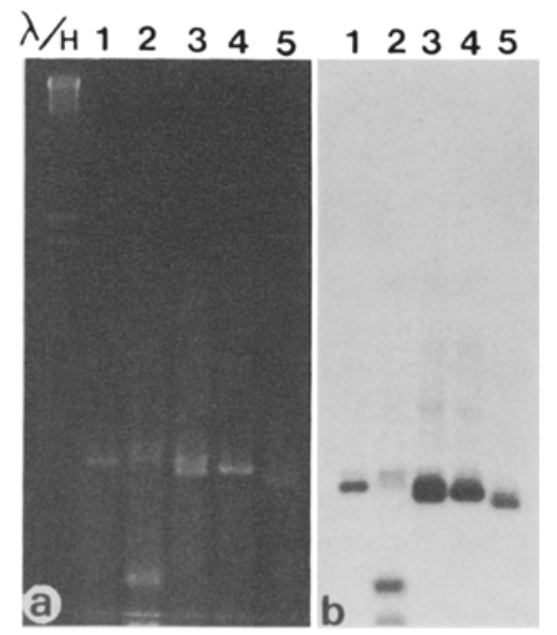

Fig. 6. Restriction mapping of the amplified PCR product (a) and Southern blot analyses (b) from purified peripheral blood. Lane 1, no enzyme added; lane 2, SphI digestion; lane 3, EcoRI digestion; lane 4, Pst I digestion; lane 5, EcoRI and Pst I double digestion.

with the probe (Fig. 5b). Physical mapping was further performed to characterize the Y-chromosomal amplified product (Fig. 6). SphI digestion resulted in 404-bp and 316-bp fragments (lane 2); EcoRI digestion showed 689-bp and 31-bp fragments (lane 3); PstI gave 697-bp and 23-bp fragments (lane 4); EcoRI and Pst I double digestion resulted in 666-bp, 31-bp, and 23-bp fragments (lane 5). The restricted fragments were hybridized with the probe (Fig. 6b). Smaller fragments run out 
of the current gel shown in Fig. 6. These results were in accord with the complete nucleotide sequence of the target region of pY-80 (Fig. 3).

\section{PCR of DNA from various samples}

Buccal epithelial cells, hair root, and urinary epithelial cells were all suitable for Y-chromosomal PCR (data not shown). None of the discrete amplified products could be seen in female samples. All of the 719-bp amplified products derived from male specimens tested hybridized with the probe, whereas no hybridizable products were detected in the female samples. Dried blood spots on a filter paper were stored at $4^{\circ} \mathrm{C}$ for periods up to 4 weeks and then DNA was extracted. The 719-bp fragment was synthesized exclusively in male DNA after the PCR. Amniotic fluid cell samples from three pregnant women were analyzed with the $Y$ chromosomal FCR. All had the Y chromosome. These findings were also confirmed by chromusomal analyses.

\section{DISCUSSION}

The pY-80 clone described here was derived from the short arm of the $\mathrm{Y}$ chromosome, and had no homology with previously sequenced $\mathrm{Y}$ specific clones (Cooke, et al., 1984; Nakahori et al., 1986; Arnemann et al., 1987; Ellis et al., 1989; Sinclair et al., 1990). It is yet to be determined whether or not the clone is identical with previously identified, but yet to be sequenced, DNA clones at the short arm of the $\mathrm{Y}$ chrcmosome. Its male specificity was confirmed by Southern tlot analysis and the PCR amplification. In view of the shortage of sequenced Y-specific probe, it would be useful for various purposes that include PCR amplification.

The results of our preliminary tests indicated that the clone is useful for sex determination in samples of various sources, including mouth wash products, plucked hair, urine, dried blood spots on a filter paper, and amniotic fluid cells.

Further analysis is being carried out of the clone's localization in relation to other previously identified DNA clones in the short arm of the Y chromosome.

Acknowledgments We are indebted to Drs. H. Kanno and Y. Kikuchi for their technical help and suggestion.

This work was supported in part by a Grant-in-Aid (No. 01570536) from the Ministry of Education, Science and Culture of Japan.

\section{REFERENCES}

Arnemann, J., Eppen, J.T., Cooke, H.J., Sauermann, U., Engel, W. and Schmidtke, J. 1987. A human Y-chromosomal DNA sequence expressed in testicular tissue. Nucleic Acids Res. 15: 8713-8724.

Cooke, H.J., Brown, W.A.R. and Rappold, G.A. 1984 . Closely related sequences on human X and $\mathrm{Y}$ chromosomes outside the pairing region. Nature 311: 259-261.

Ellis, N.A., Goodfellow, P.J., Pym, B., Smith, M., Palmer, M., Frischauf, A.-M. and Goodfellow, 
P.N. 1989. The pseudoautosomal boundary in man is defined by an Alu repeat sequence inserted on the $\mathrm{Y}$ chromosome. Nature 337: 81-84.

Nakahori, Y., Mitani, K., Yamada, M. and Nakagome, Y. 1986. A human Y-chromosome specific repeated DNA family (DYZ1) consists of a tandem array of pentanucleotides. Nucleic Acids Res. 14: 7569-7580.

Sinclair, A.H., Berta, P., Palmer, M.S., Hawkins, J.R., Griffiths, B.L., Smith, M.J., Foster, J.W., Frischauf, A.-M., Lovell-Badge, R. and Goodfellow, P.N. 1990. A gene from the human sex-determining region encodes a protein with homology to a conserved DNA-binding motif. Nature 346: 240-244.

Tsukahara, M. and Kajii, T. 1985. Replication of X chromosomes in complete moles. Hum. Genet. 71: 7-10.

Wyman, A.R. and White R. 1980. A highly polymorphic locus in human DNA. Proc. Natl. Acad. Sci. U.S.A. 77: 6754-6758.

Zabel, B.U., Naylor, S.L., Sakaguchi, A.Y., Bell, G.I. and Shows, T.B. 1983. High-resolution chromosomal Iocalization of human genes for amylase, proopiomelanocortin, somatostatin, and a DNA fragment (D3S1) by in situ hybridization. Proc. Natl. Acad. Sci. U.S.A. 80: 69326936. 